\title{
Ezetimibe Improves Rosuvastatin Effects on Inflammation and Vascular Endothelial Function in Acute Coronary Syndrome Patients Undergoing PCI
}

\author{
Changqing Sun, Wuyang Zheng, Ling Liang, Zuheng Liu, Wenchao Sun, and Rong Tang \\ Department of Cardiology, The First Affiliated Hospital of Xiamen University, Xiamen 361003, China \\ Correspondence should be addressed to Rong Tang; tang.rong@163.com
}

Received 3 May 2021; Revised 26 July 2021; Accepted 18 August 2021; Published 8 September 2021

Academic Editor: Leonardo De Luca

Copyright (C) 2021 Changqing Sun et al. This is an open access article distributed under the Creative Commons Attribution License, which permits unrestricted use, distribution, and reproduction in any medium, provided the original work is properly cited.

\begin{abstract}
Background. Little is known of the acute effects of ezetimibe in patients with acute coronary syndrome (ACS) undergoing PCI. We investigated whether ezetimibe improves inflammation and vascular endothelial function in patients with ACS undergoing PCI. Methods. We randomized 171 patients with ACS undergoing PCI to receive ezetimibe $10 \mathrm{mg} /$ day plus rosuvastatin $20 \mathrm{mg} / \mathrm{day}$ (combination group, $n=81$ ) versus rosuvastatin $20 \mathrm{mg} /$ day (rosuvastatin group, $n=90$ ). Lipid profile, type II secretory phospholipase A2 (sPLA2-IIa), interleukin-1 $\beta$ (IL-1 $\beta$ ), vascular cell adhesion molecule-1 (VCAM-1), and intercellular cell adhesion molecule-1 (ICAM-1) were measured at baseline and after 7 days. Three months after PCI, clinical outcomes were examined. Result. The levels of sPLA2-IIa and IL- $1 \beta$ reduced significantly in both groups, but more when ezetimibe and rosuvastatin were coadministered (sPLA2-IIa: $6.16 \pm 2.67$ vs. $7.42 \pm 3.53 \mathrm{ng} / \mathrm{ml}, p=0.01$; IL- $1 \beta: 37.39 \pm 26.25$ vs. $48.98 \pm 32.26 \mathrm{pg} / \mathrm{ml}, p=0.01$ ). A significant rise of VCAM-1 and ICAM-1 was observed on day 7 after PCI in the both groups, but was less in the combination group (VCAM-1: $918.28 \pm 235.31$ vs. $988.54 \pm 194.41 \mathrm{ng} / \mathrm{ml}, p=0.03$; ICAM-1: $213.01 \pm 100.15$ vs. $246.88 \pm 105.71 \mathrm{ng} / \mathrm{ml}$, $p=0.03$ ). Patients in the combination versus rosuvastatin group appeared to suffer from less major adverse events. Periprocedural therapy of ezetimibe improves rosuvastatin effects on proinflammatory responses and endothelial function associated with ACS patients undergoing PCI. This trial is registered with https://clinicaltrials.gov/ct2/show/ChiCTR-IPR-17012219 (Chinese Clinical Trial Registry, http://www.chictr.org.cn on 02/08/2017).
\end{abstract}

\section{Introduction}

Statins, inhibitors of 3-hydroxy-3-methylglutaryl coenzyme A reductase, are well-established first-line agents for the primary and secondary prevention of atherosclerotic cardiovascular disease $[1,2]$, mainly due to their powerful effect on lowering low-density lipoprotein cholesterol (LDL-C) levels [3] and anti-inflammatory property [4, 5]. Deposition of LDL-C in the arterial endothelium and the inflammation activated by it play a key role in the pathogenesis of atherosclerosis [6]. Ezetimibe blocks absorption of dietary and biliary cholesterol from the gut by inhibiting its target molecule, Niemann-Pick C1-like 1 (NPC1L1), a cholesterol transporter enriched in the apical membrane of small intestine absorptive enterocytes where it mediates extracellular cholesterol transport across the brush border membrane $[7,8]$. Ezetimibe can further decrease LDL-C levels by $6 \%-$ $25 \%$ when coadministered with a statin [9].

A recent study evaluated the effects of dual LDL-Clowering therapy with ezetimibe-statin in patients with acute coronary syndrome (ACS) on IVUS-derived coronary atherosclerosis and showed stronger coronary plaque regression during a 9-12-month of follow-up, compared with statin monotherapy [10]. In another trial (the IMPROVE-IT trial), combination therapy with ezetimibe plus statin versus statin alone improved clinical outcomes of ACS patients, including cardiovascular death, myocardial infarction, stroke, unstable angina leading to hospitalization and coronary revascularization $\geq 30$ days during a median 6-year follow-up [11]. Additionally, several studies have 
demonstrated that combination therapy with ezetimibe plus statin for 6-8 weeks reduces inflammation, aortic stiffness, and oxidative stress and improves endothelial function in patients with acute or stable coronary artery disease and rheumatoid arthritis [12-14]. However, the acute effects of coadministration with ezetimibe plus statin in patients with ACS undergoing percutaneous coronary intervention (PCI) remained unknown. Moreover, the potential mechanisms underlying the clinical benefits of ezetimibe and statin coadministration in patients with ACS have not been explored. We hypothesized that the benefits of combination therapy may be partly related to its effects on inflammation and endothelial function in addition to further cholesterollowering. This study was designed to evaluate whether periprocedural combination therapy of ezetimibe plus statin improves postprocedural levels of blood parameters of inflammation (sPLA2-IIa and IL-1 $\beta$ ) and vascular endothelial dysfunction (VCAM-1and ICAM-1) in ACS patients undergoing selective PCI.

\section{Materials and Methods}

2.1. Patients. This study is a single-center, prospective, randomized, and controlled trial conducted and reported in accordance with the requirements of the CONSORT statement. Patients who were hospitalized for ACS within the preceding 10 days, with acute myocardial infarction (MI) with or without electrocardiographic ST-segment elevation or high-risk unstable angina (UA), and underwent PCI based on the recommendations of the Chinese Guidelines [15] were eligible for inclusion. Subjects were excluded if having (1) established malignant tumor; (2) infectious or immunological disease; or (3) severe pulmonary, hepatic, or renal diseases. Additionally, participants were excluded if they were taking statins or ezetimibe prior to enrollment into this study. A total of 171 patients were randomly assigned to either rosuvastatin $20 \mathrm{mg} /$ day, or the combination of ezetimibe $(10 \mathrm{mg} /$ day $)$ plus rosuvastatin $(20 \mathrm{mg} /$ day $)$, in addition to standard ACS therapy. The study was performed according to the Helsinki Declaration and was approved by the Medical Research Ethics Committee of the Southwest Hospital, Army Medical University (approval code no: 2017-38). Written informed consent was obtained from the participant enrolled in this study.

2.2. Blood Analyses. Blood samples for measurements of parameters of inflammation and vascular endothelial dysfunction were drawn at admission and on day 7 after PCI and immediately centrifuged at $4^{\circ} \mathrm{C}$ for $5 \mathrm{~min}$ at $3000 \times \mathrm{g}$. The plasma was collected and stored at $-70^{\circ} \mathrm{C}$ until analysis. Commercially available enzyme-linked immunosorbent assays (ELISA) kits were used to determine plasma levels of sPLA2-IIa (Cayman Chemicals, Ann Arbor, MI, USA), IL$1 \beta$, VCAM-1, and ICAM-1 (R\&D Systems, Minneapolis, MNUSA). All measurements were performed according to the manufacturers' instructions. Each sample was tested in triplicate, and the mean of the triplicate was used for statistical analysis.
2.3. Clinical Follow-Up. After a 7-day treatment with rosuvastatin alone or coadministration of ezetimibe and rosuvastatin, all patients received daily $20 \mathrm{mg}$ rosuvastatin for the secondary prevention of cardiovascular events. A clinical follow-up 3 months post-PCI was done by office visits or telephone calls in all participants. The effects of rosuvastatin alone or combination of ezetimibe and rosuvastatin on middle-term clinical outcomes in these patients were documented, including the occurrences of CV death, nonfatal MI, UA requiring hospitalization, coronary revascularization, and nonfatal stroke [11].

2.4. Statistical Analyses. Statistical analyses were performed with SPSS 18.0 for Windows (SPSS, Chicago, IL, USA). Continuous variables were presented as means \pm standard deviation (SD), and categorical data were expressed as numbers and frequencies. Two-tailed independent $t$ tests and Mann-Whitney $U$ tests were used to compare parameters between the two groups. Frequency was tested with the chi-squared test. A 2 -tailed $p$ value $<0.05$ was considered statistically significant.

\section{Result}

3.1. Characteristics of the Study Subjects. A total of 171 patients with ACS were enrolled in this trial. One patient died 8 days after PCI because of interventricular septum rupture and 2 patients had pneumonia 2 days after PCI. One patient had gastrointestinal hemorrhage 24 hours after PCI and received blood transfusion. All 171 patients (90 patients in the rosuvastatin group and 81 patients in the combination group) completed the 7-day trial according to the protocol. Clinical characteristics and medical therapies in the two treatment groups were not significantly different at baseline (Table 1). The two groups were also similar in coronary anatomy, procedural characteristics, diameter, and length of implanted stents (Table 2). Ezetimibe- or statin-induced toxicity, such as persistent transaminase elevation, myopathy, or rhabdomyolysis, was not observed in patients enrolled in this study.

3.2. Plasma Levels of Lipids. At baseline, the plasma levels of total cholesterol (TC), LDL-C, high-density lipoprotein cholesterol (HDL-C), and triglyceride (TG) in the two treatment groups were not significantly different. On day 7 after PCI, a greater reduction in plasma levels of TC and LDL-C was observed in the combination group versus statin monotherapy (TC: $14.1 \%$ vs. $5.9 \%, p=0.02$; LDL-C: $15.6 \%$ vs. $6.1 \%, p=0.04)$. HDL-C and TG levels were not significantly reduced in each group after 7 days of drug treatment post-PCI and showed no statistical difference between the two groups after the treatment (Table 3).

3.3. Plasma Levels of Markers for Proinflammatory Responses and Vascular Endothelial Dysfunction. Plasma levels of proinflammatory markers sPLA2-IIa and IL-1 $\beta$ as well as vascular endothelial dysfunction makers VCAM-1 and 
TABLE 1: Baseline characteristics of the study subjects.

\begin{tabular}{|c|c|c|c|}
\hline & Rosuvastatin $(n=90)$ & Ezetimibe/rosuvastatin $(n=81)$ & $p$ value \\
\hline Male, $n(\%)$ & $63(70.0 \%)$ & $55(67.9 \%)$ & 0.77 \\
\hline Age (years) & $64.08 \pm 10.45$ & $61.74 \pm 8.78$ & 0.12 \\
\hline BMI $\left(\mathrm{kg} / \mathrm{m}^{2}\right)$ & $24.36 \pm 3.18$ & $24.24 \pm 3.49$ & 0.82 \\
\hline Current smokers, $n(\%)$ & $44(48.9 \%)$ & $34(42.0 \%)$ & 0.37 \\
\hline Diabetes mellitus, $n(\%)$ & $30(33.3)$ & $29(35.8)$ & 0.74 \\
\hline Hypertension, $n(\%)$ & $50(55.5)$ & $41(50.6)$ & 0.52 \\
\hline Dyslipidemia, $n(\%)$ & $37(41.1)$ & $23(28.4)$ & 0.08 \\
\hline LVEF (\%) & $59.00 \pm 7.47$ & $60.17 \pm 7.87$ & 0.32 \\
\hline \multicolumn{4}{|l|}{ Presentation of ACS } \\
\hline STEMI, $n(\%)$ & $24(26.7)$ & $16(19.8)$ & 0.29 \\
\hline NSTEMI, $n(\%)$ & $13(14.4)$ & $6(7.4)$ & 0.14 \\
\hline $\mathrm{UA}, n(\%)$ & $53(58.9)$ & $59(72.8)$ & 0.06 \\
\hline \multicolumn{4}{|l|}{ Previous medications } \\
\hline Aspirin, $n(\%)$ & $10(11.1)$ & $7(8.6)$ & 0.59 \\
\hline Clopidogrel, $n(\%)$ & $5(5.6)$ & $3(3.7)$ & 0.57 \\
\hline Beta-blockers, $n(\%)$ & $10(11.1)$ & $5(6.2)$ & 0.25 \\
\hline ACE inhibitors, $n(\%)$ & $14(15.6)$ & $9(11.1)$ & 0.40 \\
\hline
\end{tabular}

Values are presented as $n$ (\%) or mean \pm standard deviation. BMI, body mass index; LVEF, left ventricular ejection fraction; ACS, acute coronary syndrome; STEMI, ST-segment elevation myocardial infarction; NSTEMI, none ST-segment elevation myocardial infarction; UA, unstable angina; ACE, angiotensinconverting enzyme.

TABLE 2: Procedural characteristics.

\begin{tabular}{|c|c|c|c|}
\hline & Rosuvastatin $(n=90)$ & Ezetimibe/rosuvastatin $(n=81)$ & $p$ value \\
\hline \multicolumn{4}{|l|}{ Vessel treated } \\
\hline Left anterior descending & $52(57.8)$ & $48(59.3)$ & 0.84 \\
\hline Left circumflex & $25(27.8)$ & $16(19.8)$ & 0.22 \\
\hline Right coronary artery & $33(36.7)$ & $27(33.3)$ & 0.65 \\
\hline \multicolumn{4}{|l|}{ Type of intervention } \\
\hline Balloon only & $8(8.9)$ & $5(6.2)$ & 0.50 \\
\hline Stent & $82(91.1)$ & $76(93.8)$ & 0.50 \\
\hline No. of stents per patient & $1.43 \pm 0.63$ & $1.26 \pm 0.57$ & 0.10 \\
\hline Stent diameter $(\mathrm{mm})$ & $2.98 \pm 0.34$ & $3.00 \pm 0.34$ & 0.83 \\
\hline Total stent length (mm) & $30.37 \pm 15.38$ & $26.04 \pm 15.29$ & 0.08 \\
\hline Direct stenting, $n(\%)$ & $14(17.1)$ & $10(13.2)$ & 0.49 \\
\hline No. of predilatations & $68(82.9)$ & $66(86.8)$ & 0.49 \\
\hline
\end{tabular}

Values are presented as $n(\%)$ or mean \pm standard deviation.

TABLE 3: Values of blood lipids at baseline and 7 days after PCI.

\begin{tabular}{|c|c|c|c|}
\hline & Rosuvastatin $(n=90)$ & Ezetimibe/rosuvastatin $(n=81)$ & $p$ value \\
\hline \multicolumn{4}{|l|}{ TC (mg/dl) } \\
\hline Baseline & $177.50 \pm 53.36$ & $175.95 \pm 48.34$ & 0.83 \\
\hline Day 7 & $167.05 \pm 49.50$ & $151.20 \pm 37.12$ & 0.02 \\
\hline$p$ value & $<0.01$ & $<0.01$ & \\
\hline \multicolumn{4}{|c|}{ LDL-C (mg/dl) } \\
\hline Baseline & $113.69 \pm 40.99$ & $114.08 \pm 32.87$ & 0.97 \\
\hline Day 7 & $106.73 \pm 37.90$ & $96.29 \pm 22.82$ & 0.04 \\
\hline$p$ value & $<0.01$ & $<0.01$ & \\
\hline \multicolumn{4}{|c|}{ HDL-C (mg/dl) } \\
\hline Baseline & $39.44 \pm 10.44$ & $42.15 \pm 10.44$ & 0.13 \\
\hline Day 7 & $39.06 \pm 9.28$ & $40.22 \pm 8.89$ & 0.41 \\
\hline$p$ value & 0.29 & 0.13 & \\
\hline \multicolumn{4}{|l|}{$\mathrm{TG}(\mathrm{mg} / \mathrm{dl})$} \\
\hline Baseline & $183.29 \pm 130.16$ & $173.55 \pm 122.19$ & 0.47 \\
\hline Day 7 & $171.78 \pm 146.10$ & $168.24 \pm 143.44$ & 0.78 \\
\hline$p$ value & 0.09 & 0.27 & \\
\hline
\end{tabular}

Values are presented as mean \pm standard deviation. 
ICAM-1 were similar in the two groups at the time of randomization. At the 7 th day postprocedure, the plasma levels of sPLA2-IIa reduced significantly from baseline in both groups (rosuvastatin: from $8.32 \pm 3.86 \mathrm{ng} / \mathrm{ml}$ to $7.42 \pm 3.53 \mathrm{ng} / \mathrm{ml}, p=0.02$; combination: from $9.11 \pm$ $4.35 \mathrm{ng} / \mathrm{ml}$ to $6.16 \pm 2.67 \mathrm{ng} / \mathrm{ml}, p<0.01$ ), and this decrease was significantly lower in the combination versus rosuvastatin group $(6.16 \pm 2.67$ vs. $7.42 \pm 3.53 \mathrm{ng} / \mathrm{ml}, p=0.01)$. Similarly, IL- $1 \beta$ levels decreased significantly at the $7^{\text {th }}$ day after the procedure in both groups (rosuvastatin: from $62.99 \pm 35.01 \mathrm{pg} / \mathrm{ml}$ to $48.98 \pm 32.26 \mathrm{pg} / \mathrm{ml}, \quad p<0.01$; combination: from $67.95 \pm 31.56 \mathrm{pg} / \mathrm{ml}$ to $37.39 \pm 26.25 \mathrm{pg} / \mathrm{ml}$, $p<0.01)$. Coadministration of ezetimibe with rosuvastatin resulted in a significant lower level of IL- $1 \beta$ than rosuvastatin alone $(37.39 \pm 26.25$ vs. $48.98 \pm 32.26 \mathrm{pg} / \mathrm{ml}, p=0.01)$ (Figure 1).

Unlike plasma sPLA2-IIa and IL-1 $\beta$ levels, a significant rise in plasma VCAM-1 and ICAM-1 levels was observed at the 7 th day after PCI in the rosuvastatin group (VCAM1: from $876.32 \pm 184.78 \mathrm{ng} / \mathrm{ml}$ to $988.54 \pm 194.41 \mathrm{ng} / \mathrm{ml}$, $p<0.01$; ICAM-1: from $201.65 \pm 104.04 \mathrm{ng} / \mathrm{ml}$ to $246.88 \pm 105.71 \mathrm{ng} / \mathrm{ml}, p<0.01)$ and in the combination group (VCAM-1: from $843.31 \pm 211.87 \mathrm{ng} / \mathrm{ml}$ to $918.28 \pm$ $235.31 \mathrm{ng} / \mathrm{ml}, p<0.01$; ICAM-1: from $204.64 \pm 107.22 \mathrm{ng} / \mathrm{ml}$ to $213.01 \pm 100.15 \mathrm{ng} / \mathrm{ml}, p=0.02$ ). However, combination therapy of rosuvastatin and ezetimibe led to significantly lower levels of VCAM-1 (918.28 \pm 235.31 vs. 988.54 \pm $194.41 \mathrm{ng} / \mathrm{ml}, p=0.03)$ and ICAM-1 (213.01 \pm 100.15 vs. $246.88 \pm 105.71 \mathrm{ng} / \mathrm{ml}, p=0.03)$, compared to rosuvastatin monotherapy (Figure 1).

3.4. Clinical Follow-Up Outcomes. To determine whether the 7 days of postprocedure combination therapy of ezetimibe and rosuvastatin has any impact on middle-term clinical outcomes, we followed up the patients for 3 months. The patients in the combination group appeared to suffer from less major adverse events including CV death, nonfatal MI, UA requiring hospitalization, or coronary revascularization during this period, but no statistical difference was observed (Table 4).

\section{Discussion}

This study demonstrates that the coadministration of ezetimibe and rosuvastatin in patients with ACS leads to greater reduction in the proinflammatory factors as reflected in plasma concentrations of IL- $1 \beta$ and sPLA2-IIa at the 7 th day after PCI, as compared to rosuvastatin treatment alone. The combined therapy versus rosuvastatin alone is better in protecting the coronary angioplasty-induced increase in plasma ICAM-1 and VCAM-1 levels. These findings suggest that combined therapy relative to monotherapy may be a better option for cardiovascular protection in ACS patients undergoing PCI. Additionally, our results show that the 7day postprocedure coadministration of ezetimibe and rosuvastatin in patients with ACS undergoing PCI improves the major adverse events, including the occurrences of $\mathrm{CV}$ death, nonfatal MI, UA, and coronary revascularization, during a three-month follow-up, though no statistical significance is obtained likely due to the short duration of the follow-up. To the best of our knowledge, this trial is the first to document the effects of acute application of aggressive cholesterol-lowering therapy (i.e., ezetimibe plus rosuvastatin) in the periprocedural period of PCI in patients with ACS.

Interleukin $1 \beta$ (IL-1 $\beta$ ) is a proinflammatory cytokine [16] that has been widely documented to play critical roles in nearly all stages of atherosclerosis from early plaque formation to the destabilization and rupture of advanced lesions [17]. Cholesterol in circulation is taken up by monocyte-derived macrophages [18-20], inducing inflammation by stimulating the caspase-1-activating NLRP3 inflammasome, which results in cleavage and secretion of IL$1 \beta$ and other proinflammatory cytokines [21]. Estruch et al. reported that LDL induces priming and inflammasome activation leading to IL- $1 \beta$ release in human monocytes and macrophages [22]. Therefore, IL-1 $\beta$ levels may be influenced by lipid-lowering therapy. In our study, ezetimibe/rosuvastatin coadministration relative to rosuvastatin monotherapy achieved a greater reduction in plasma LDL-C and IL-1 $\beta$ levels. Similar to our study, Moutzouri et al. [23] reported that high simvastatin dose or the combination of a low-dose simvastatin with ezetimibe reduces IL-1 $\beta$ production in monocytes of hypercholesterolemic patients. Another prospective and randomized study in CAD patients demonstrated that atorvastatin markedly downregulates the expression of NLRP3 inflammasome [24], which activates proteolytic enzyme caspase- 1 to cleave pro-IL- $1 \beta$ producing the active mature IL- $1 \beta$ peptide. However, a recent study showed no association between statin use and IL- $1 \beta$ levels in a healthy population [25]. The apparent discrepancy among these studies including ours may be explained by the different complications of the subjects and that high-risk subjects versus normal subjects probably benefit more.

In the present study, periprocedural coadministration of ezetimibe and rosuvastatin relative to rosuvastatin alone significantly reduces plasma sPLA2-IIa levels in ACS patients undergoing PCI. It has been shown that rosuvastatin and ezetimibe combination inhibits the cytokines induced by the action of IL- $1 \beta[26,27]$. Accumulating evidence from basic research and clinical trials has demonstrated that sPLA2-IIa plays an important role in the pathogenesis of atherosclerosis and the instability of the atherosclerotic plaque [28, 29]. Thus, our findings may have important clinical implications.

Recruitment and adhesion of monocytes to the arterial endothelial lining is one of the earliest detectable events during atherogenesis [30]. Endothelial activation molecules (e.g., ICAM-1 and VCAM-1) play crucial roles in the cascade of cell interactions that mediate extravasation and migration of inflammatory cells into the vascular endothelium [31]. Therefore, circulating endothelial activation molecules are regarded as surrogate markers of low-grade vascular inflammation and endothelial dysfunction. Numerous studies have shown that coronary angioplasty is followed by a transient increase in circulating adhesion molecule levels within 24 hours after the procedure [32-34] due to local 

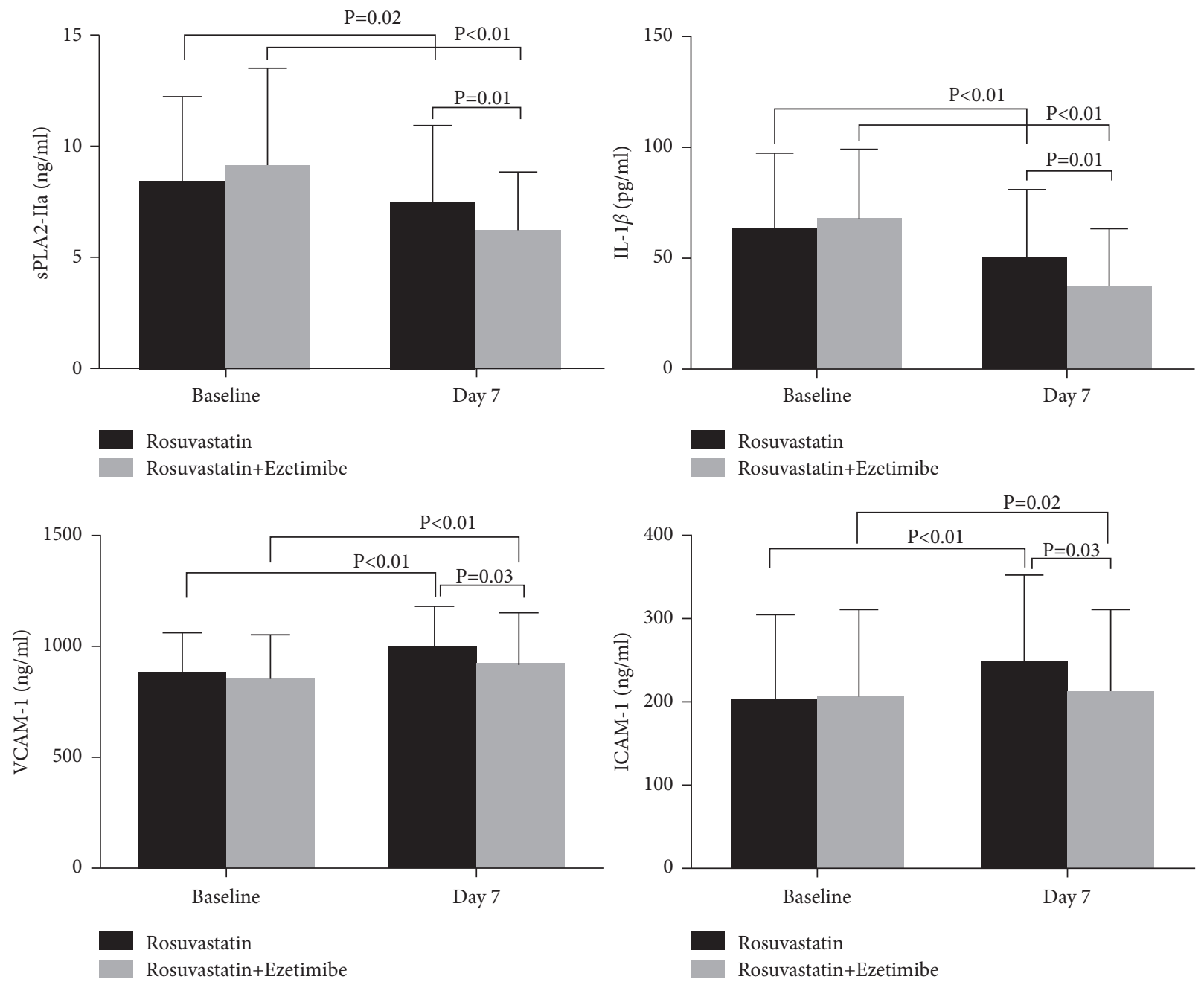

Figure 1: Plasma levels of sPLA2-IIa, IL-1 $\beta$, VCAM-1, and ICAM-1 in patients with ACS on baseline and 7th day after PCI.

TABle 4: Clinical follow-up outcomes within the three-month after PCI.

\begin{tabular}{lccc}
\hline & Rosuvastatin $(n=90)$ & Ezetimibe/rosuvastatin $(n=81)$ & $p$ value \\
\hline CV death, $n(\%)$ & $3(3.3)$ & $1(1.2)$ & 0.62 \\
Nonfatal MI, $n(\%)$ & $4(4.4)$ & $3(3.7)$ & 0.72 \\
UA, $n(\%)$ & $10(11.1)$ & $8(9.9)$ & 0.81 \\
Revascularization, $n(\%)$ & $7(7.8)$ & $5(6.2)$ & 0.68 \\
Nonfatal stroke, $n(\%)$ & 0 & 0 & - \\
\hline
\end{tabular}

Data are summarized as $n$ (\%). CV, cardiovascular; MI, myocardial infarction; UA, unstable angina.

endothelial damage [34]. Our results confirm these findings by showing that adhesion molecule levels are increased at the 7 th day after percutaneous intervention. We did not detect significant correlations between changes in adhesion molecules and LDL-C following lipid-lowering therapy in the two groups, suggesting that improvement in endothelial function may not depend on drugs' lipid-lowering effects. An early study in patients undergoing PCI showed that reduction of procedural myocardial injury after 7-day pretreatment with atorvastatin was paralleled by concomitant attenuation of postprocedural increase of adhesion molecule levels [32]. These findings may explain the protective effects of statin on myocardial damage during coronary intervention observed in the ARMDYDA trial [35]. In a recent trial [36], the benefit of periprocedural loading doses of statins among ACS patients was observed in patients undergoing PCI. Because the reduction of MACE observed in this study occurred early after statin initiation, the mechanism behind this potential effect is likely the statins' pleiotropic effects. However, no previous studies have evaluated the effects of short-term treatment with ezetimibe and a statin on adhesion molecule levels in ACS patients undergoing PCI. Our finding that combined therapy relative to rosuvastatin monotherapy is more 
effective in attenuating the PCI-stimulated increase in blood adhesion molecules highlighting the possibility that ezetimibe and rosuvastatin coadministration versus rosuvastatin alone may promote better clinical outcomes in patients with ACS undergoing PCI. Indeed, we observed reduced cardiovascular events in the combination group, though our study may be underpowered to obtain statistically significant differences on clinical endpoints between the two groups due to the small sample size and the relatively short observation duration.

\section{Conclusion}

The present study shows that coadministration of ezetimibe and rosuvastatin relative to rosuvastatin monotherapy is more effective in improving plasma markers of proinflammatory responses and vascular endothelial dysfunction in ACS patients undergoing PCI. Our findings may improve clinical practice in these patients. In addition, our observations may help with further clarification of the mechanisms underlying the potential clinical benefits provided by double lipid-lowering therapy, as well as the pharmacological basis of the pleiotropic protection of combination therapy.

\section{Data Availability}

The datasets used to support the findings of this study are available from the corresponding author upon request.

\section{Consent}

Not Applicable.

\section{Conflicts of Interest}

The authors declare that they have no conflicts of interest.

\section{Authors' Contributions}

C.S. and R.T. conceived and revised the manuscript and led the team to accomplish the study. C.S., W.Z., L.L., and Z.L. performed the study. C.S. and W.S. analyzed the data and wrote the article. All authors reviewed the manuscript. Changqing Sun and Wuyang Zheng equally contributed to this study.

\section{Acknowledgments}

This work was supported by Xiamen Science and Technology Project (3502Z20209018).

\section{References}

[1] M. B. Mortensen, S. Afzal, B. G. Nordestgaard, and E. Falk, "Primary prevention with statins," Journal of the American College of Cardiology, vol. 66, no. 24, pp. 2699-2709, 2015.

[2] N. J. Stone, "Statins in secondary prevention," Journal of the American College of Cardiology, vol. 69, no. 22, pp. 2707-2709, 2017.
[3] S. J. Nicholls, G. Brandrup-Wognsen, M. Palmer, and P. J. Barter, "Meta-analysis of comparative efficacy of increasing dose of atorvastatin versus rosuvastatin versus simvastatin on lowering levels of atherogenic lipids (from VOYAGER)," The American Journal of Cardiology, vol. 105, no. 1, pp. 69-76, 2010.

[4] P. A. McCullough, "The antiinflammatory effects of statins," New England Journal of Medicine, vol. 345, no. 16, pp. 1209-1211, 2001.

[5] D. J. Lefer, "Statins as potent antiinflammatory drugs," Circulation, vol. 106, no. 16, pp. 2041-2042, 2002.

[6] J. A. Berliner, M. Navab, A. M. Fogelman et al., "Atherosclerosis: basic mechanisms," Circulation, vol. 91, no. 9, pp. 2488-2496, 1995.

[7] S. W. Altmann, H. R. Davis, L. J. Zhu et al., "Niemann-pick C1 like 1 protein is critical for intestinal cholesterol absorption," Science, vol. 303, no. 5661, pp. 1201-1204, 2004.

[8] J. L. Betters and L. Yu, "NPC1L1 and cholesterol transport," FEBS Letters, vol. 584, no. 13, pp. 2740-2747, 2010.

[9] D. P. Mikhailidis, G. C. Sibbring, C. M. Ballantyne, G. M. Davies, and A. L. Catapano, "Meta-analysis of the cholesterol-lowering effect of ezetimibe added to ongoing statin therapy," Current Medical Research and Opinion, vol. 23, no. 8, pp. 2009-2026, 2007.

[10] K. Tsujita, K. Yamanaga, N. Komura et al., "Lipid profile associated with coronary plaque regression in patients with acute coronary syndrome: subanalysis of PRECISE-IVUS trial," Atherosclerosis, vol. 251, pp. 367-372, 2016.

[11] S. A. Murphy, C. P. Cannon, M. A. Blazing et al., "Reduction in total cardiovascular events with ezetimibe/simvastatin post-acute coronary syndrome," Journal of the American College of Cardiology, vol. 67, no. 4, pp. 353-361, 2016.

[12] M. Azar, E. Valentin, G. Badaoui, R. Kassab, A. Sarkis, and R. R. Azar, "Comparison of the effects of combination atorvastatin $(40 \mathrm{mg})+$ ezetimibe $(10 \mathrm{mg})$ versus atorvastatin $(40 \mathrm{mg})$ alone on secretory phospholipase A2 activity in patients with stable coronary artery disease or coronary artery disease equivalent," The American Journal of Cardiology, vol. 107, no. 11, pp. 1571-1574, 2011.

[13] K. M. Mäki-Petäjä, A. D. Booth, F. C. Hall et al., "Ezetimibe and simvastatin reduce inflammation, disease activity, and aortic stiffness and improve endothelial function in rheumatoid arthritis," Journal of the American College of Cardiology, vol. 50, no. 9, pp. 852-858, 2007.

[14] A. Undas, A. Machnik, D. P. Potaczek, E. Wypasek, K. Zmudka, and W. Tracz, "Ezetimibe combined with simvastatin compared with simvastatin alone results in a greater suppression of oxidative stress and enhanced fibrinolysis in patients after acute coronary events," Journal of Cardiovascular Pharmacology, vol. 58, no. 2, pp. 167-172, 2011.

[15] "Chinese guideline for percutaneous coronary intervention (2016)," Zhonghua Xinxueguanbing Zazhi, vol. 44, pp. 382400, 2016.

[16] C. A. Dinarello, "Blocking IL-1 in systemic inflammation," Journal of Experimental Medicine, vol. 201, no. 9, pp. 13551359, 2005.

[17] S. Apostolakis, K. Vogiatzi, E. Krambovitis, and D. Spandidos, "IL-1 cytokines in cardiovascular disease: diagnostic, prognostic and therapeutic implications," Cardiovascular and Hematological Agents in Medicinal Chemistry, vol. 6, no. 2, pp. 150-158, 2008.

[18] S. M. Lessner, H. L. Prado, E. K. Waller, and Z. S. Galis, "Atherosclerotic lesions grow through recruitment and proliferation of circulating monocytes in a murine model," 
American Journal of Pathology, vol. 160, no. 6, pp. 2145-2155, 2002.

[19] F. K. Swirski, M. J. Pittet, M. F. Kircher et al., "Monocyte accumulation in mouse atherogenesis is progressive and proportional to extent of disease," Proceedings of the National Academy of Sciences, vol. 103, no. 27, pp. 10340-10345, 2006.

[20] L. Landsman, L. Bar-On, A. Zernecke et al., "CX3CR1 is required for monocyte homeostasis and atherogenesis by promoting cell survival," Blood, vol. 113, no. 4, pp. 963-972, 2009.

[21] P. Duewell, H. Kono, K. J. Rayner et al., "NLRP3 inflammasomes are required for atherogenesis and activated by cholesterol crystals," Nature, vol. 464, no. 7293, pp. 1357-1361, 2010.

[22] M. Estruch, K. Rajamäki, J. L. Sanchez-Quesada et al., "Electronegative LDL induces priming and inflammasome activation leading to IL- $1 \beta$ release in human monocytes and macrophages," Biochimica et Biophysica Acta (BBA)-Molecular and Cell Biology of Lipids, vol. 1851, no. 11, pp. 1442-1449, 2015.

[23] E. Moutzouri, C. C. Tellis, K. Rousouli et al., "Effect of simvastatin or its combination with ezetimibe on toll-like receptor expression and lipopolysaccharide-induced cytokine production in monocytes of hypercholesterolemic patients," Atherosclerosis, vol. 225, no. 2, pp. 381-387, 2012.

[24] M. Satoh, T. Tabuchi, T. Itoh, and M. Nakamura, "NLRP3 inflammasome activation in coronary artery disease: results from prospective and randomized study of treatment with atorvastatin or rosuvastatin," Clinical Science, vol. 126, no. 3, pp. 233-241, 2014.

[25] T. Lyngdoh, P. Vollenweider, G. Waeber, and P. MarquesVidal, "Association of statins with inflammatory cytokines: a population-based colaus study," Atherosclerosis, vol. 219, no. 1, pp. 253-258, 2011.

[26] M. Schwemmer, H. Aho, and J.-B. Michel, "Interleukin-1 $\beta$ induced type IIA secreted phospholipase A2 gene expression and extracellular activity in rat vascular endothelial cells," Tissue and Cell, vol. 33, no. 3, pp. 233-240, 2001.

[27] A. Jaulmes, S. Thierry, B. Janvier et al., "Activation of sPLA2IIA and PGE2 production by high mobility group protein $\mathrm{B} 1$ in vascular smooth muscle cells sensitized by IL-1 $\beta$," The FASEB Journal, vol. 20, no. 10, pp. 1727-1729, 2006.

[28] M. Kimura-Matsumoto, Y. Ishikawa, K. Komiyama et al., "Expression of secretory phospholipase A2s in human atherosclerosis development," Atherosclerosis, vol. 196, no. 1, pp. 81-91, 2008.

[29] P. Liu, Y. H. Li, W. C. Tsai et al., "Prognostic value and the changes of plasma levels of secretory type II phospholipase A2 in patients with coronary artery disease undergoing percutaneous coronary intervention," European Heart Journal, vol. 24, no. 20, pp. 1824-1832, 2003.

[30] R. Ross, "The pathogenesis of atherosclerosis: a perspective for the 1990s," Nature, vol. 362, no. 6423, pp. 801-809, 1993.

[31] D. Vestweber and J. E. Blanks, "Mechanisms that regulate the function of the selectins and their ligands," Physiological Reviews, vol. 79, no. 1, pp. 181-213, 1999.

[32] G. Patti, M. Chello, V. Pasceri et al., "Protection from procedural myocardial injury by atorvastatin is associated with lower levels of adhesion molecules after percutaneous coronary intervention," Journal of the American College of Cardiology, vol. 48, no. 8, pp. 1560-1566, 2006.

[33] G. A. A. Ferns, L. A. Forster, J. C. William et al., "Effect of vitamin $\mathrm{E}$ supplementation on circulating cell adhesion molecules pre- and post-coronary angioplasty," Annals of
Clinical Biochemistry: International Journal of Laboratory Medicine, vol. 37, no. 5, pp. 649-654, 2000.

[34] T. Siminiak, J. F. Dye, R. M. Egdell, R. More, H. Wysocki, and D. J. Sheridan, "The release of soluble adhesion molecules ICAM-1 and E-selectin after acute myocardial infarction and following coronary angioplasty," International Journal of Cardiology, vol. 61, no. 2, pp. 113-118, 1997.

[35] V. Pasceri, G. Patti, A. Nusca, C. Pristipino, G. Richichi, and G. Di Sciascio, "Randomized trial of atorvastatin for reduction of myocardial damage during coronary intervention," Circulation, vol. 110, no. 6, pp. 674-678, 2004.

[36] O. Berwanger, E. V. Santucci, P. G. M. de Barros E Silva et al., "Effect of loading dose of atorvastatin prior to planned percutaneous coronary intervention on major adverse cardiovascular events in acute coronary syndrome," Journal of the American Medical Association, vol. 319, no. 13, pp. 1331-1340, 2018. 